\title{
Escala de expectativas parentales acerca de la prosocialidad de los hijos: construcción y análisis psicométricos preliminares
}

\author{
Jael Vargas Rubilar¹, Viviana Noemí Lemos² y Cinthia Balabanian ${ }^{3}$
}

\begin{abstract}
RESUMEN
Introducción: Las expectativas parentales son las creencias de los padres respecto al desempeño de sus hijos en algún área específica. En este estudio se consideró, de modo particular, las expectativas de los padres acerca de la conducta prosocial de sus hijos. La conducta prosocial es definida como aquella llevada a cabo voluntariamente con el fin de beneficiar a otros. Objetivo: Desarrollar un instrumento que evalúe las expectativas parentales acerca de la prosocialidad de los hijos y evaluar preliminarmente sus propiedades psicométricas. Método: Inicialmente, se redactó un pool de 50 ítems, los cuales fueron sometidos a la evaluación de 5 jueces expertos. La versión ajustada fue administrada a una muestra piloto de 20 padres, quienes evaluaron la comprensión y claridad de los reactivos utilizados. Posteriormente la escala revisada fue administrada a 486 padres de ambos sexos (edad: $M=38.6$; $D E=9.7$ ) de las provincias de Córdoba, Mendoza, Santa Fe y Entre Ríos (Argentina). Se calcularon los estadísticos descriptivos para cada ítem, el poder discriminativo y el índice de homogeneidad corregido. Resultados: Todos los items resultaron discriminativos $(p<.001)$. A partir de una submuestra de 267 casos, se realizó un AFE que permitió una depuración inicial de items. Con una submuestra de 219 casos se corroboró la estructura unidimensional del instrumento mediante un AFC. La escala final quedó conformada por 28 reactivos, operacionalizados a través de un factor, el cual explicó un $61 \%$ de la variancia total. La consistencia interna de la escala fue satisfactoria $(\omega=.97)$. Conclusión: Los análisis realizados hasta el momento indican adecuadas propiedades psicométricas por lo que se infiere que la escala permitirá valorar las expectativas parentales acerca de la conducta prosocial de manera satisfactoria en el contexto en que fue estudiada.
\end{abstract}

Palabras clave: expectativas parentales, conducta prosocial, estudio psicométrico, niños.

\section{Scale of parental expectations about children's prosociality: preliminary construction and psychometric analysis}

\begin{abstract}
Introduction: Parental expectations refer to those beliefs parents have regarding their children's performance in a specific area. In particular, the present study examines parents' expectations about their children's prosocial behavior. Prosocial behavior is
\end{abstract}

\footnotetext{
${ }^{1}$ Centro Interdisciplinario de Investigaciones en Psicologia Matemática y Experimental (CIIPME). Consejo Nacional de Investigaciones Científicas y Técnicas (CONICET). Universidad Adventista del Plata, Argentina; psicojael@gmail.com; https://orcid.org/0000-0002-6689-6845

${ }^{2}$ Centro Interdisciplinario de Investigaciones en Psicología Matemática y Experimental (CIIPME). Consejo Nacional de Investigaciones Cientificas y Técnicas (CONICET). Universidad Adventista del Plata, Argentina; viviananoemilemos@gmail.com; https://orcid.org/0000-0002-8855-2293

3 Centro Interdisciplinario de Investigaciones en Psicologia Matemática y Experimental (CIIPME). Consejo Nacional de Investigaciones Cientificas y Técnicas (CONICET). Universidad Adventista del Plata, Argentina; cinthia.balabanian@gmail.com; https://orcid.org/0000-0002-4189-0623
} 
defined as voluntary performance carried out so as to benefit others. Objective: Developing an instrument in order to evaluate parental expectations about their children's prosocial behavior and conducting a preliminary assessment of its psychometric properties. Method: Initially, a pool of 50 items submitted to the assessment of five expert judges was drafted. The adjusted version was administered to a pilot sample of 20 parents who evaluated the understanding and clarity of the reactives used. Subsequently, the revised scale was administered to 486 parents of both sexes $(M=39.4$; $S D=5.85$ ) from the provinces of Córdoba and Entre Ríos (Argentina). Descriptive statistics for each item, their discriminative power and the homogeneity index corrected by item was evaluated. Results: All items were discriminative $(p<.001)$. From a subsample of 267 cases, EFA was conducted in order to obtain an initial item refinement. The one-dimensional structure of the instrument was corroborated by CFA using a subsample of 219 cases. The final scale was composed of 28 items, operationalized through a single factor which explained $61 \%$ of the total variance. The internal consistency of the scale was satisfactory $(\omega=.97)$. Conclusion: The analysis carried out so far show adequate psychometric properties, which suggests that the scale will successfully enable to assess parental expectations about prosocial behavior within the context of study.

Keywords: parental expectations, prosocial behavior, psychometric study, children.

La influencia del contexto familiar, tanto a nivel intrapersonal como social, ha tenido un gran desarrollo en la investigación psicológica. Si bien los agentes socializadores y los ámbitos de socialización pueden ser diversos (escuela, barrio, etc.), el énfasis de la bibliografia ha sido puesto en el ambiente familiar y, especialmente, en el rol de los padres. Así, el interés por el estudio de la parentalidad o función de crianza de los padres se ha incrementado desde los años setenta hasta la actualidad (Vargas Rubilar \& Richaud de Minzi, 2018).

El ejercicio de una parentalidad positiva o adecuada contribuye de manera relevante en el desarrollo de los recursos socioemocionales de los hijos (Vargas Rubilar \& Richaud de Minzi, 2018), incluyendo competencias del área emocional y social (Squires, Bricker, \& Twombly, 2013). Es decir, capacidades que permiten identificar, regular y expresar emociones en función de una meta específica, (i.e. emocional) y relacionarse de una manera adecuada con los demás (i.e., sociales).

En esta dirección, en un estudio reciente (Vargas Rubilar, Oros \& Lemos, 2016) se realizó una revisión de las investigaciones argentinas que estudiaron el impacto de la parentalidad (i.e., apego y estilos parentales) sobre las emociones positivas (i.e., alegria, serenidad, simpatia, satisfacción y gratitud) y negativas (i.e., depresión y soledad) y sobre las conductas sociales positivas (i.e., asertividad y prosocialidad) y las negativas (i.e., agresión, arrogancia y ansiedad social). Se encontró que los estilos de crianza más positivos y el apego seguro favorecen el desarrollo de emociones y conducta sociales positivas y disminuyen las emociones y comportamientos negativos.

Particularmente, en lo que respecta al rol de la parentalidad en la prosocialidad infantil, algunos trabajos han informado que los estilos parentales tienen una importante influencia en el comportamiento prosocial infantil (Mestre Escrivá, Tur, Samper, Nácher, \& Cortés, 2007; Richaud de Minzi, 2009; Richaud de Minzi, Lemos, \& Mesurado, 2011). Por otra parte, otros estudios han encontrado incidencia del apego en la prosocialidad de los adolescentes (e.g., Balabanian, Lemos, \& Vargas Rubilar, 2015; SánchezQueija \& Oliva, 2003; Oliva Delgado, 2011; Ortiz, et al., 1993). Asimismo, una investigación llevada a cabo por Wyatt y Carlo (2002) encontró que los 
adolescentes que esperaban una reacción materna y paterna más positiva frente a su conducta prosocial informaban estar más implicados en actividades prosociales.

Según Baumrind (1978), la socialización parental es un proceso iniciado por los adultos por medio del cual los niños adquieren los hábitos y los valores congruentes con la adaptación a la propia cultura, a través de la educación y la imitación. Por lo tanto, los padres tienen un incomparable efecto sobre la personalidad, y la competencia de sus hijos. En este estudio pionero sobre estilos parentales, se identificó un estilo que ella llamó armónico, el cual mostró que los padres no necesariamente ejercen control directo, sino que el niño trata de intuir y hacer lo que sus padres quieren y esperan de él. Esto podria indicar la particular importancia que tienen las expectativas de los padres en las emociones y comportamientos de los hijos.

Bornstein (2007), por su parte, señala que los padres tienen una clara propensión a guiar a los niños hacia las metas que ellos consideran importantes. Aunque existen diversas definiciones de las "expectativas parentales", la mayoría de los autores (e.g. Goldenberg, Gallimore, Reese, \& Garnier, 2001; Yamamoto \& Holloway, 2010) coincide en que estas son las creencias realistas de los padres respecto al logro futuro de sus hijos en algún área específica (e.g., calificaciones, nivel de escolaridad, etc.). La mayoría de los estudios revisados se han centrado en analizar el rol de las expectativas de los padres en el rendimiento escolar o académico de los hijos (Bandura, Barbaranelli, Caprara, \& Pastorelli, 1996; Yamamoto \& Holloway, 2010). Muchos de estos trabajos operacionalizan las expectativas de los padres preguntándoles, "hasta qué punto" o "qué tan lejos" (how far) creen que su hijo puede llegar en su desempeño, por ejemplo, con respecto al cursado durante ese año escolar. En lo que respecta al área afectiva, otro estudio (Oishi \& Sullivan, 2005), realizado en diferentes culturas, mostró una relación positiva entre las expectativas parentales con el bienestar subjetivo y la autoestima de los hijos.

A pesar de que las aspiraciones y expectativas de los padres, para algunos autores, son constructos conceptualmente diferentes, suelen usarse indistintamente (e.g., Juang \& Silbereisen, 2002). Incluso, en algunos estudios, los investigadores utilizan las aspiraciones y expectativas de los padres por separado (e.g., Carpenter, 2008), y otros, combinadas en una sola medida (e.g., Bandura et al., 1996). Las expectativas de los padres se pueden contrastar con las aspiraciones de los mismos, en que, por lo general, estas últimas se refieren a deseos u objetivos no realistas que los padres tienen acerca del futuro de sus hijos (Yamamoto \& Holloway, 2010).

En el presente estudio consideraremos particularmente las expectativas parentales acerca de la conducta prosocial de los hijos, última la que entendemos como aquellos comportamientos que son llevados a cabo voluntariamente con el fin de beneficiar a otros (Mestre Escrivá, Samper, Tur, Cortés, \& Nácher, 2006; Roche Olivar, 1995). Debido a las consecuencias positivas que la conducta prosocial conlleva en la sociedad, la promoción de este tipo de comportamientos se considera clave para prevenir los crecientes problemas de agresividad (Lemos \& Richaud de Minzi, 2014; Redondo Pacheco, Rueda Rueda, \& Amado Vega, 2013; Richaud de Minzi, 2009; Richaud de Minzi et al., 2013).

Clásicamente, el constructo conducta prosocial ha sido conceptualizado como multidimensional, puesto que abarca un gran número de diferentes 
tipos de comportamientos positivos destinados a beneficiar a otros (Roche Olivar, 1998). Entre dichos comportamientos se encuentran la ayuda física directa, la ayuda verbal, los actos de cooperación, la revalorización positiva del otro, el confort y consuelo ante un momento de angustia, el rescate, la presencia positiva y unidad, etc. (Caprara, Steca, Zelli, \& Capanna, 2005; Dunfield, 2014; Eisenberg, Fabes, \& Spinrad, 2006; Grant \& Dutton, 2012; Lemos \& Richaud de Minzi,2014; Mestre Escrivá, et al., 2006; Roche Olivar, 1995; Samper García, 2014; Warneken \& Tomasello, 2009). Sin embargo, en la actualidad la bibliografia propone un número más reducido de factores generales que englobarian las diferentes conductas prosociales (Auné \& Attorresi, 2017).

\section{Evaluación de las expectativas parentales}

En lo que respecta a instrumentos de evaluación, Wyatt y Carlo (2002) desarrollaron una escala para evaluar cómo los adolescentes esperan que sus padres reaccionen frente a sus diferentes conductas. Dicha escala analiza la reacción parental que los adolescentes esperan en relación con sus conductas antisociales y conductas prosociales. No obstante, esta prueba solo permite obtener información respecto a la reacción parental esperada desde la perspectiva de los hijos y no valora las expectativas de los padres respecto a las conductas prosociales de sus hijos. Por otra parte, tal como se ha mencionado anteriormente, existe un buen número de pruebas para evaluar las expectativas parentales respecto de la escolarización y el rendimiento académico de los hijos (e.g. Bandura, et al., 1996; Yamamoto \& Holloway, 2010). Sin embargo, no se han encontrado pruebas que permitan valorar este aspecto de la parentalidad en relación con conductas sociales positivas.

En sintesis, se estima que son escasos los estudios acerca del rol que juegan las expectativas parentales en la prosocialidad de sus hijos. Hasta el momento, no se han encontrado investigaciones en nuestro contexto que aborden específicamente esta temática en padres de niños en edad escolar. En este marco, el principal objetivo de este trabajo fue diseñar una escala que valore las expectativas parentales acerca de la conducta prosocial de los hijos $\mathrm{y}$ analizar preliminarmente su funcionamiento psicométrico en padres de niños escolarizados.

\section{MÉTODO}

Se realizó un estudio de tipo instrumental, ya que este estuvo orientado al diseño de una prueba $\mathrm{y}$ al estudio de sus propiedades psicométricas (Montero \& León, 2007).

\section{Participantes}

Participaron del estudio 5 jueces expertos y 20 padres que conformaron una primera muestra piloto. Posteriormente, se trabajó con una muestra conformada por 486 padres de niños escolarizados $(74.8 \%$ mujeres y $25.2 \%$ hombres; edad: $M=39.4 ; D E=5.85$ ), pertenecientes a diferentes familias. En lo que respecta a la escolaridad, el $15 \%$ tenía estudios secundarios incompletos, el $26 \%$ secundarios completos, el 56\% había cursado estudios terciarios o universitarios y el resto de posgrado. Los participantes eran padres y madres de niños de 9 a 12 años $(M=10.59 ; D E=1.21)$ que asistían a escuelas de clase media públicas y privadas de las provincias de Córdoba 
(47\%), Mendoza (11\%), Santa Fe (32\%) y Entre Ríos (10\%), Argentina. Para la selección de los participantes se realizó un muestreo no probabilístico por accesibilidad o conveniencia (Otzen \& Manterola, 2017).

\section{Instrumentos}

Se administró una encuesta sobre datos sociodemográficos junto con la escala que se iba a estudiar, en la que se solicitaban datos concernientes a edad, sexo, lugar de residencia y escolaridad.

La escala, desarrollada y administrada para su estudio psicométrico, estuvo conformada por 50 items. La consigna establecía que el padre o madre debía indicar, en una escala de 1 a 10, cuánto esperaría que su hijo tenga determinado comportamiento o actitud prosocial, en donde 1 implicaria un menor grado de expectativa y 10 el mayor grado de expectativa de que se manifieste dicho comportamiento. Este rango de posibles opciones de respuesta fue escogido dado que es una escala de uso cotidiano en nuestro contexto escolar-académico. Asimismo, algunos estudios han señalado que un formato Likert de más anclajes podría mejorar la fiabilidad y favorecer una estructura factorial más clara y precisa, mejorando de este modo la medición del constructo (Tomas \& Oliver, 1998).

\section{Procedimiento}

\section{Formulación de los ítems}

Los diferentes items se redactaron a partir de un criterio racionalempírico-teórico, considerando comportamientos prosociales factibles de expresarse en diferentes ámbitos en este rango etario. Fueron tenidos en cuenta la Escala de Conducta Prosocial para niños de 9 a 12 años de Lemos (2015) y los aportes de Warneken y Tomasello (2009) en cuanto a los tipos de conductas prosociales que podría incluir el instrumento: confortar, consolar y cuidar, compartir, ayudar física y verbalmente, revalorizar positivamente a los demás y ser una presencia positiva. Además, se consideró el aporte de otros autores que han evaluado el comportamiento prosocial en población adolescente y adulta (Balabanian \& Lemos, 2018; Caprara, et al., 2005; Roche Olivar, 1995).

En la tabla 1 pueden observarse los ítems de la escala inicial, en la que se indica su ámbito de manifestación y el tipo específico de conducta prosocial operacionalizada. 
Tabla 1.

Ítems incluidos en la escala según los tipos conducta prosocial y ámbitos en que se manifiestan.

\begin{tabular}{|c|c|c|}
\hline Ítems de la escala inicial & Ámbito & Dimensión \\
\hline $\begin{array}{l}\text { 1. Defienda a algún compañero ante alguna } \\
\text { injusticia. }\end{array}$ & Escolar & Presencia positiva/unidad \\
\hline $\begin{array}{l}\text { 2. Se ofrezca a ayudar en las tareas de la } \\
\text { casa. }\end{array}$ & Familiar & Cooperación \\
\hline $\begin{array}{l}\text { 3. Ayude a mantener limpia y ordenada su } \\
\text { pieza. }\end{array}$ & Familiar & Cooperación \\
\hline 4. Trate bien a sus hermanos. & Familiar & Presencia positiva/unidad \\
\hline 5. Ayude a sus abuelos en algo que necesiten. & Familiar & Ayuda \\
\hline 6. Cuide a su hermano/a. & Familiar & Cuidar/confortar/consolar \\
\hline $\begin{array}{l}\text { 7. Explique a algún compañero un tema que le } \\
\text { cueste entender. }\end{array}$ & Escolar & Ayuda \\
\hline $\begin{array}{l}\text { 8. Ayude a algún compañero a terminar la } \\
\text { tarea. }\end{array}$ & Escolar & Ayuda \\
\hline $\begin{array}{l}\text { 9. Explique a otros compañeros las tareas } \\
\text { escolares. }\end{array}$ & Escolar & Ayuda \\
\hline 10. Ayude a limpiar y acomodar el aula. & Escolar & Cooperación \\
\hline $\begin{array}{l}\text { 11. Regale a alguien que necesite algunos de } \\
\text { sus juguetes o ropa. }\end{array}$ & No especificado & Dar y compartir \\
\hline $\begin{array}{l}\text { 12. Ayude a la seño a llevar las cosas cuando } \\
\text { está muy cargada. }\end{array}$ & Escolar & Ayuda \\
\hline $\begin{array}{l}\text { 13.Done su ropa en buen estado, no solo lo } \\
\text { que le sobra. }\end{array}$ & No especificado & Dar y compartir \\
\hline $\begin{array}{l}\text { 14.Comparta juegos con sus hermanos/primos. } \\
\text { 15.Comparta la merienda con sus compañeros. } \\
\text { 16. Comparta sus útiles. }\end{array}$ & $\begin{array}{l}\text { Familiar } \\
\text { Escolar } \\
\text { Escolar }\end{array}$ & $\begin{array}{l}\text { Dar y compartir } \\
\text { Dar y compartir } \\
\text { Dar y compartir }\end{array}$ \\
\hline $\begin{array}{l}\text { 17.Acerque las tareas de la escuela a algún } \\
\text { compañero que está enfermo. }\end{array}$ & Escolar & Ayudar \\
\hline $\begin{array}{l}\text { 18.Preste sus libros a los compañeros que no } \\
\text { tienen. }\end{array}$ & Escolar & Dar y compartir \\
\hline $\begin{array}{l}\text { 19. Le levante el ánimo a alguien de la familia } \\
\text { que se sienta mal. }\end{array}$ & Familiar & Confortar/consolar/cuidar \\
\hline $\begin{array}{l}\text { 20.Consuele a algún compañero que esté } \\
\text { triste. }\end{array}$ & Escolar & Confortar/consolar/cuidar \\
\hline $\begin{array}{l}\text { 21.Se ofrezca a acompañar a algún compañero } \\
\text { que se siente mal. }\end{array}$ & Escolar & Confortar/consolar/cuidar \\
\hline $\begin{array}{l}\text { 22.Pida disculpas cuando fuera necesario. } \\
\text { 23. Sea buen compañero. }\end{array}$ & $\begin{array}{l}\text { No especificado } \\
\text { Escolar }\end{array}$ & $\begin{array}{l}\text { Presencia positiva/unidad } \\
\text { Presencia positiva/unidad }\end{array}$ \\
\hline $\begin{array}{l}\text { 24. Se dé cuenta de las cosas lindas de los } \\
\text { demás y se las diga. }\end{array}$ & No especificado & Revalorización positiva del otro \\
\hline $\begin{array}{l}\text { 25. Defienda a sus amigos/familiares. } \\
\text { 26.Diga cumplidos a sus hermanos. } \\
\text { 27. Integre a otros a su grupo de amigos. } \\
\text { 28.Valore las ideas de sus compañeros. } \\
\text { 29. Sea comprensivo con los demás. }\end{array}$ & $\begin{array}{l}\text { No especificado/Familiar } \\
\text { Familiar } \\
\text { No especificado } \\
\text { Escolar } \\
\text { No especificado }\end{array}$ & $\begin{array}{c}\text { Presencia positiva/unidad } \\
\text { Revalorización positiva del otro } \\
\text { Presencia positiva/unidad } \\
\text { Revalorización positiva del otro } \\
\text { Presencia positiva/unidad }\end{array}$ \\
\hline $\begin{array}{l}\text { 30.Participe en un grupo o club de } \\
\text { beneficencia. }\end{array}$ & No especificado & Ayudar \\
\hline $\begin{array}{l}\text { 31. Tenga una actitud de colaboración en las } \\
\text { tareas grupales. }\end{array}$ & Escolar & Cooperación \\
\hline $\begin{array}{l}\text { 32.Reconozca las cualidades de sus } \\
\text { compañeros. }\end{array}$ & Escolar & Revalorización positiva del otro \\
\hline $\begin{array}{l}\text { 33. Colabore haciendo silencio cuando la } \\
\text { maestra habla. }\end{array}$ & Escolar & Cooperación \\
\hline 34.Acepte a los demás sin juzgarlos. & No especificado & Presencia positiva/unidad \\
\hline $\begin{array}{l}\text { 35. Escuche con atención su opinión y la del } \\
\text { resto de los miembros de la familia. }\end{array}$ & Familiar & Presencia positiva/unidad \\
\hline 36. Colabore con las tareas del hogar. & Familiar & Cooperación \\
\hline $\begin{array}{l}\text { 37. Responda de buena manera cuando se le } \\
\text { pide algo. }\end{array}$ & No especificado & Presencia positiva \\
\hline $\begin{array}{l}\text { 38. Felicite a otro cuando se saca una buena } \\
\text { nota. }\end{array}$ & Escolar & Revalorización positiva \\
\hline
\end{tabular}


Tabla 1.

Ítems incluidos en la escala según los tipos conducta prosocial y ámbitos en que se manifiestan (continuación).

\begin{tabular}{|c|c|c|}
\hline Ítems de la escala inicial & Ámbito & Dimensión \\
\hline \multirow{10}{*}{$\begin{array}{l}\text { 39. Sea amigable. } \\
\text { 40. Trate bien a los demás. } \\
\text { 41. Disfrute de hacer regalos. } \\
\text { 42. No diga cosas que pueden molestar a } \\
\text { otros. } \\
\text { 43. Sea bueno con todos por igual. } \\
\text { 44. Sea bueno con los animales. } \\
\text { 45. Sepa dar las gracias cuando alguien hace } \\
\text { algo bueno para él. } \\
\text { 46. Sorprenda a otros con cosas lindas. } \\
\text { 47. Sea conciliador. } \\
\text { 48. Esté disponible cuando un amigo lo } \\
\text { necesite. }\end{array}$} & No especificado & Presencia positiva/unidad \\
\hline & No especificado & Presencia positiva/unidad \\
\hline & No especificado & Dar y compartir \\
\hline & No especificado & Presencia positiva/unidad \\
\hline & No especificado & Presencia positiva \\
\hline & No especificado & Cuidar \\
\hline & No especificado & Revalorización positiva \\
\hline & No especificado & Presencia positiva \\
\hline & No especificado & Presencia positiva \\
\hline & No especificado & Presencia positiva \\
\hline $\begin{array}{l}\text { 49. Que no participe en burlas hacia algún } \\
\text { compañero. }\end{array}$ & No especificado & Presencia positiva \\
\hline 50. Felicite a otros cuando hacen algo bien. & No especificado & Revalorización positiva \\
\hline
\end{tabular}

\section{Procedimientos seguidos para el estudio de los ítems y de la escala}

Para el estudio y ajuste de la escala inicial se utilizó la siguiente secuencia metodológica: (a) revisión de jueces expertos, (b) aplicación a una muestra piloto y posteriores ajustes, (c) aplicación a una muestra más extensa; (d) análisis descriptivos por ítem (media, desvío, asimetría y curtosis), (e) análisis del poder discriminativo de los items e indice de homogeneidad corregido, (f) a continuación la muestra total fue dividida de forma aleatoria en dos grupos: por un lado, el $55 \%$ de los casos $(n=267)$ conformó una submuestra que se utilizó para llevar a cabo un AFE con el software FACTOR 8 (Lorenzo-Seva \& Ferrando, 2013) y el análisis de la consistencia interna a través del coeficiente Omega (McDonald, 1999; Ventura-León \& Caycho Rodríguez, 2017), (g) el $45 \%$ restante de los sujetos $(n=219)$ conformaron la segunda submuestra, a partir de la cual se calculó un AFC, utilizando el software LISREL 8.8 (Jöreskog \& Sörborn, 1993); finalmente, a partir de los pesos cargas factoriales, se estimó la fiabilidad compuesta y la variancia media extractada.

\section{Procedimientos seguidos en la recolección de datos}

Los padres que participaron del estudio fueron contactados, en todos los casos, a través de la institución escolar a la que asistian sus hijos. Previo a realizar la evaluación, se explicaron los objetivos de la investigación junto con los recaudos éticos de confidencialidad y anonimato de los datos de los participantes. Se solicitó el consentimiento informado, explicitando el carácter voluntario de su participación. La evaluación de la prueba se realizó en el marco de un proyecto de investigación en el que participaron tanto padres como hijos. Por esta razón, se le solicitó a uno de los padres (o tutor) que respondiera la escala de expectativas parentales únicamente en función de su hijo o hija que era parte del estudio. Las administraciones fueron individuales y estuvieron a cargo de psicólogos entrenados para dicha evaluación. 


\section{RESULTADOS}

\section{Revisión de los jueces expertos}

Los reactivos fueron sometidos a un proceso de depuración primaria. En primer lugar, fueron evaluados por cinco jueces expertos (i.e. psicólogos y psicopedagogos con formación en investigación) sobre la base de los siguientes criterios: (a) congruencia entre la definición operacional y la definición teórica del constructo medido, (b) grado de relevancia como indicador del constructo, (c) pertinencia en función de los aspectos sintácticos, semánticos y nivel de comprensión para la población a la que está dirigida el instrumento. Las sugerencias de los jueces en esta instancia posibilitaron algunas reformulaciones menores de los items.

\section{Evaluación de la muestra piloto}

Posteriormente, se realizó una prueba piloto con un grupo de 20 padres, quienes aportaron sus opiniones para mejorar la claridad y comprensión de las afirmaciones. A partir de sus comentarios, se modificaron algunas palabras o frases que resultaron poco familiares para los evaluados.

\section{Análisis de ítems: discriminación y homogeneidad}

Se calcularon los estadísticos descriptivos y los valores de asimetría y curtosis con sus correspondientes errores típicos para cada item (ver tabla 2). Dado que muchos de los items obtuvieron valores de asimetría y curtosis por encima de los guarismos \pm 2 , recomendados para realizar análisis paramétricos (Bandalos \& Finney, 2010; Muthén \& Kaplan, 1985), el poder discriminativo de los ítems fue evaluado a partir de una comparación de rangos entre los sujetos ubicados por encima del percentil 75 con los ubicados por debajo del 25, utilizando la prueba $U$ de Mann-Whitney. Como puede observarse en la tabla 3, todos los ítems resultaron discriminativos. También fue calculado el indice de homogeneidad corregido (IHc) y, como puede verse en la tabla 2, todos los reactivos obtuvieron indices aceptables, con valores mayores a .30 (Kline, 2005). Este resultado corrobora la capacidad de discriminación de los reactivos y su aporte al constructo evaluado. 
Tabla 2

Estadísticos descriptivos para el conjunto de 50 ítems.

\begin{tabular}{|c|c|c|c|c|c|c|c|}
\hline & \multirow{2}{*}{$M$} & \multirow{2}{*}{$D E$} & \multicolumn{2}{|c|}{ Asimetría } & \multicolumn{2}{|c|}{ Curtosis } & \multirow{2}{*}{ IHC } \\
\hline & & & Estadístico & Error estándar & Estadístico & Error estándar & \\
\hline Ítem 1 & 8.56 & 1.72 & -1.81 & 0.12 & 4.51 & 0.23 & .457 \\
\hline Ítem 2 & 8.07 & 1.99 & -1.15 & 0.12 & 1.09 & 0.23 & .488 \\
\hline Ítem 3 & 8.20 & 1.99 & -1.42 & 0.12 & 1.99 & 0.23 & .497 \\
\hline Ítem 4 & 8.86 & 1.61 & -2.08 & 0.12 & 5.40 & 0.23 & .584 \\
\hline Ítem 5 & 8.99 & 1.55 & -2.36 & 0.12 & 6.77 & 0.23 & .558 \\
\hline Ítem 6 & 8.54 & 1.83 & -1.85 & 0.12 & 3.93 & 0.23 & .418 \\
\hline Ítem 7 & 8.53 & 1.59 & -1.41 & 0.12 & 2.49 & 0.23 & .654 \\
\hline Ítem 8 & 8.10 & 1.95 & -1.47 & 0.12 & 2.60 & 0.23 & .587 \\
\hline Ítem 9 & 8.23 & 1.80 & -1.28 & 0.12 & 2.04 & 0.23 & .590 \\
\hline Ítem 10 & 8.29 & 1.86 & -1.57 & 0.12 & 2.99 & 0.23 & .591 \\
\hline Ítem 11 & 9.18 & 1.30 & -2.34 & 0.12 & 7.53 & 0.23 & .636 \\
\hline Ítem 12 & 8.76 & 1.67 & -2.12 & 0.12 & 5.72 & 0.23 & .593 \\
\hline Ítem 13 & 8.99 & 1.56 & -2.29 & 0.12 & 6.42 & 0.23 & .618 \\
\hline Ítem 14 & 9.10 & 1.26 & -1.84 & 0.12 & 4.36 & 0.23 & .607 \\
\hline Ítem 15 & 8.72 & 1.59 & -1.88 & 0.12 & 5.04 & 0.23 & .605 \\
\hline Ítem 16 & 8.53 & 1.72 & -1.40 & 0.12 & 2.15 & 0.23 & .581 \\
\hline Ítem 17 & 8.51 & 1.84 & -1.81 & 0.12 & 3.81 & 0.23 & .622 \\
\hline Ítem 18 & 8.75 & 1.64 & -1.94 & 0.12 & 4.59 & 0.23 & .634 \\
\hline Ítem 19 & 8.94 & 1.48 & -1.83 & 0.12 & 4.17 & 0.23 & .611 \\
\hline Ítem 20 & 8.88 & 1.39 & -1.62 & 0.12 & 3.41 & 0.23 & .742 \\
\hline Ítem 21 & 8.78 & 1.58 & -1.92 & 0.12 & 4.62 & 0.23 & .701 \\
\hline Ítem 22 & 9.11 & 1.56 & -2.46 & 0.12 & 7.04 & 0.23 & .649 \\
\hline Ítem 23 & 9.30 & 1.24 & -2.56 & 0.12 & 8.97 & 0.23 & .748 \\
\hline Ítem 24 & 8.74 & 1.53 & -1.78 & 0.12 & 4.22 & 0.23 & .736 \\
\hline Ítem 25 & 9.04 & 1.30 & -1.87 & 0.12 & 4.76 & 0.23 & .621 \\
\hline Ítem 26 & 8.33 & 1.67 & -1.38 & 0.12 & 2.59 & 0.23 & .567 \\
\hline Ítem 27 & 8.71 & 1.52 & -1.86 & 0.12 & 4.97 & 0.23 & .707 \\
\hline Ítem 28 & 8.76 & 1.40 & -1.43 & 0.12 & 2.98 & 0.23 & .775 \\
\hline Ítem 29 & 8.75 & 1.45 & -1.72 & 0.12 & 4.24 & 0.23 & .760 \\
\hline Ítem 30 & 7.84 & 2.37 & -1.52 & 0.12 & 1.87 & 0.23 & .553 \\
\hline Ítem 31 & 8.83 & 1.30 & -1.31 & 0.12 & 1.96 & 0.23 & .725 \\
\hline Ítem 32 & 8.65 & 1.33 & -1.37 & 0.12 & 2.61 & 0.23 & .716 \\
\hline Ítem 33 & 9.04 & 1.55 & -2.08 & 0.12 & 4.70 & 0.23 & .628 \\
\hline Ítem 34 & 9.10 & 1.37 & -2.36 & 0.12 & 7.67 & 0.23 & .721 \\
\hline Ítem 35 & 8.84 & 1.46 & -1.47 & 0.12 & 2.20 & 0.23 & .677 \\
\hline Ítem 36 & 8.39 & 1.75 & -1.51 & 0.12 & 3.04 & 0.23 & .581 \\
\hline Ítem 37 & 8.72 & 1.75 & -1.97 & 0.12 & 4.54 & 0.23 & .691 \\
\hline Ítem 38 & 8.59 & 1.67 & -1.58 & 0.12 & 2.98 & 0.23 & .708 \\
\hline Ítem 39 & 9.10 & 1.44 & -2.71 & 0.12 & 10.13 & 0.23 & .710 \\
\hline Ítem 40 & 9.12 & 1.45 & -2.55 & 0.12 & 8.32 & 0.23 & .720 \\
\hline Ítem 41 & 8.71 & 1.66 & -1.88 & 0.12 & 4.61 & 0.23 & .685 \\
\hline Ítem 42 & 8.67 & 1.82 & -1.81 & 0.12 & 3.72 & 0.23 & .671 \\
\hline Ítem 43 & 8.87 & 1.52 & -1.97 & 0.12 & 5.36 & 0.23 & .664 \\
\hline Ítem 44 & 9.27 & 1.28 & -2.71 & 0.12 & 10.23 & 0.23 & .606 \\
\hline Ítem 45 & 9.27 & 1.30 & -2.69 & 0.12 & 9.53 & 0.23 & .724 \\
\hline Ítem 46 & 8.59 & 1.49 & -1.14 & 0.12 & 0.95 & 0.23 & .696 \\
\hline Ítem 47 & 8.78 & 1.44 & -1.61 & 0.12 & 4.02 & 0.23 & .725 \\
\hline Ítem 48 & 9.04 & 1.49 & -2.47 & 0.12 & 7.99 & 0.23 & .724 \\
\hline Ítem 49 & 8.95 & 2.08 & -2.58 & 0.12 & 6.40 & 0.23 & .500 \\
\hline Ítem 50 & 8.90 & 1.49 & -1.82 & 0.12 & 4.06 & 0.23 & .738 \\
\hline
\end{tabular}


Tabla 3

Poder discriminativo de los ítems.

\begin{tabular}{|c|c|c|c|c|}
\hline & Cuartil inferior & Cuartil superior & \multirow{2}{*}{$\boldsymbol{U}$} & \multirow{2}{*}{$p$} \\
\hline & Rango promedio & Rango promedio & & \\
\hline Ítem 1 & 72.61 & 149.74 & 1843.50 & $<.001$ \\
\hline Ítem 2 & 66.09 & 156.32 & 1119.50 & $<.001$ \\
\hline Ítem 3 & 66.73 & 155.68 & 1190.50 & $<.001$ \\
\hline Ítem 4 & 69.00 & 153.39 & 1442.50 & $<.001$ \\
\hline Ítem 5 & 72.73 & 149.62 & 1857.00 & $<.001$ \\
\hline Ítem 6 & 75.17 & 147.16 & 2127.50 & $<.001$ \\
\hline Ítem 7 & 61.68 & 160.77 & 630.00 & $<.001$ \\
\hline Ítem 8 & 63.11 & 159.32 & 789.50 & $<.001$ \\
\hline Ítem 9 & 63.07 & 159.36 & 785.00 & $<.001$ \\
\hline Ítem 10 & 63.49 & 158.95 & 831.00 & $<.001$ \\
\hline Ítem 11 & 72.58 & 149.77 & 1840.00 & $<.001$ \\
\hline Ítem 12 & 64.54 & 157.88 & 948.00 & $<.001$ \\
\hline Ítem 13 & 66.73 & 155.68 & 1190.50 & $<.001$ \\
\hline Ítem 14 & 68.72 & 153.66 & 1412.00 & $<.001$ \\
\hline Ítem 15 & 65.65 & 156.76 & 1071.00 & $<.001$ \\
\hline Ítem 16 & 68.19 & 154.20 & 1353.00 & $<.001$ \\
\hline Ítem 17 & 63.66 & 158.77 & 850.00 & $<.001$ \\
\hline Ítem 18 & 65.48 & 156.93 & 1052.50 & $<.001$ \\
\hline Ítem 19 & 70.04 & 152.34 & 1558.00 & $<.001$ \\
\hline Ítem 20 & 65.84 & 156.57 & 1092.50 & $<.001$ \\
\hline Ítem 21 & 65.51 & 156.90 & 1055.50 & $<.001$ \\
\hline Ítem 22 & 66.62 & 155.78 & 1179.00 & $<.001$ \\
\hline Ítem 23 & 65.91 & 156.50 & 1100.00 & $<.001$ \\
\hline Ítem 24 & 61.29 & 161.16 & 587.50 & $<.001$ \\
\hline Ítem 25 & 69.50 & 152.87 & 1499.00 & $<.001$ \\
\hline Ítem 26 & 61.85 & 160.60 & 649.50 & $<.001$ \\
\hline Ítem 27 & 61.54 & 160.91 & 615.00 & $<.001$ \\
\hline Ítem 28 & 59.35 & 163.12 & 372.00 & $<.001$ \\
\hline Ítem 29 & 60.09 & 162.37 & 454.00 & $<.001$ \\
\hline Ítem 30 & 67.27 & 155.13 & 1251.00 & $<.001$ \\
\hline Ítem 31 & 60.14 & 162.33 & 459.00 & $<.001$ \\
\hline Ítem 32 & 62.63 & 159.81 & 736.00 & $<.001$ \\
\hline Ítem 33 & 64.93 & 157.49 & 991.50 & $<.001$ \\
\hline Ítem 34 & 63.35 & 159.09 & 815.50 & $<.001$ \\
\hline Ítem 35 & 63.27 & 159.16 & 807.00 & $<.001$ \\
\hline Ítem 36 & 64.59 & 157.83 & 954.00 & $<.001$ \\
\hline Ítem 37 & 63.42 & 159.01 & 824.00 & $<.001$ \\
\hline Ítem 38 & 58.87 & 163.60 & 318.50 & $<.001$ \\
\hline Ítem 39 & 66.36 & 156.05 & 1150.00 & $<.001$ \\
\hline Ítem 40 & 65.04 & 157.38 & 1003.00 & $<.001$ \\
\hline Ítem 41 & 66.23 & 156.18 & 1135.00 & $<.001$ \\
\hline Ítem 42 & 61.64 & 160.81 & 626.00 & $<.001$ \\
\hline Ítem 43 & 62.64 & 159.80 & 737.00 & $<.001$ \\
\hline Ítem 44 & 74.65 & 147.68 & 2070.50 & $<.001$ \\
\hline Ítem 45 & 66.76 & 155.65 & 1194.00 & $<.001$ \\
\hline Ítem 46 & 62.66 & 159.78 & 739.00 & $<.001$ \\
\hline Ítem 47 & 62.50 & 159.94 & 721.50 & $<.001$ \\
\hline Ítem 48 & 66.35 & 156.05 & 1149.00 & $<.001$ \\
\hline Ítem 49 & 71.05 & 151.31 & 1670.50 & $<.001$ \\
\hline Ítem 50 & 60.03 & 162.43 & 447.50 & $<.001$ \\
\hline
\end{tabular}

\section{Análisis factorial exploratorio}

Para evaluar la estructura subyacente del instrumento, los 50 reactivos fueron sometidos a un Análisis Factorial Exploratorio (AFE). Primeramente, se evaluó la factibilidad de dicho análisis mediante el índice de adecuación 
muestral Kaiser-Meyer-Olkin (KMO) y la prueba de esfericidad de Bartlett, cuyos resultados fueron adecuados $\left(K M O=.93 ; X^{2}\right.$ de Bartlett $=11464$ (1225); $p<.001)$. Dada la distribución no normal de las variables, se partió de una matriz de correlaciones policóricas. Los factores fueron extraídos a través del método ULS robusto, dado que dicho método se considera adecuado cuando se trabaja con muestras no muy numerosas y con un número de variables elevado, además de ser recomendable para estimar el AFE desde una matriz policórica (Ferrando \& Lorenzo-Seva, 2014).

Luego del análisis y con el objetivo de reducir la escala, se incluyeron solo los items que saturaron por encima de .60 (ver tabla 4). Así, en esta etapa se eliminaron 22 de los 50 items formulados originalmente. De este modo, la escala quedó conformada por un factor con 28 reactivos. Dicho factor explicó un $61.9 \%$ de la variancia total, es decir más del $40 \%$ considerado por Carmines y Zeller (1979) para sostener una sola dimensión. Además, la razón entre el primer autovalor y el segundo era superior a 5 (12) lo cual, según Martínez-Arias, Hernández-Lloreda y Hernández-Lloreda (2006), indican que habria un factor claramente predominante.

Tabla 4.

Matriz factorial resultante de la escala propuesta.

\begin{tabular}{lc}
\hline Ítems de la escala & Cargas factoriales \\
\hline 1. Done su ropa en buen estado, no sólo lo que le sobra. (13) & .688 \\
2. Comparta juegos con sus hermanos/primos. (14) & .716 \\
3. Consuele a algún compañero que esté triste. (20) & .711 \\
4. Se ofrezca a acompañar a algún compañero que se siente mal. (21) & .679 \\
5. Pida disculpas cuando fuera necesario. (22) & .740 \\
6. Sea buen compañero. (23) & .809 \\
7. Se dé cuenta de las cosas lindas de los demás y se las diga. (24) & .769 \\
8. Integre a otros a su grupo de amigos. (27) & .729 \\
9. Valore las ideas de sus compañeros. (28) & .817 \\
10. Sea comprensivo con los demás. (29) & .787 \\
11. Tenga una actitud de colaboración en las tareas grupales. (31) & .753 \\
12. Reconozca las cualidades de sus compañeros. (32) & .766 \\
13. Colabore haciendo silencio cuando la maestra habla. (33) & .737 \\
14. Acepte a los demás sin juzgarlos. (34) & .815 \\
15. Escuche con atención su opinión y la del resto de los miembros de la familia. (35) & .730 \\
16. Responda de buena manera cuando se le pide algo. (37) & .767 \\
17. Felicite a otro cuando se saca una buena nota. (38) & .790 \\
18. Sea amigable. (39) & .800 \\
19. Trate bien a los demás. (40) & .867 \\
20. Disfrute de hacer regalos. (41) & .733 \\
21. No diga cosas que pueden molestar a otros. (42) & .813 \\
22. Sea bueno con todos por igual. (43) & .770 \\
23. Sepa dar las gracias cuando alguien hace algo bueno para él. (45) & .819 \\
24. Sorprenda a otros con cosas lindas. (46) & .744 \\
25. Sea conciliador. (47) & .820 \\
26. Esté disponible cuando un amigo lo necesite. (48) & .808 \\
27. Que no participe en burlas hacia algún compañero. (49) & .759 \\
28. Felicite a otros cuando hacen algo bien. (50) & .806 \\
\hline
\end{tabular}

Nota: Entre paréntesis se indica el número del ítem correspondiente a la versión de 50 ítems.

\section{Consistencia interna}

Para analizar la confiabilidad, particularmente el aspecto de la consistencia interna, considerando la ausencia de normalidad de los datos, se calculó el índice Omega, encontrando un valor de $\omega=.97$. 


\section{Análisis factorial confirmatorio, fiabilidad compuesta}

Finalmente, se llevó a cabo un AFC con el objetivo de evaluar si el modelo observado en el AFE se ajustaba a los datos empíricos de la segunda submuestra. Para ello, se utilizó el método de estimación de Mínimos Cuadrados no Ponderados (ULS), recomendado para variables ordinales con un tamaño muestral apropiado ( $n$ mayor o igual a 200) (Hoyle, 2012). Los indices de ajuste estimados para esta versión fueron adecuados (ver figura $1 \mathrm{y}$ tabla 5).

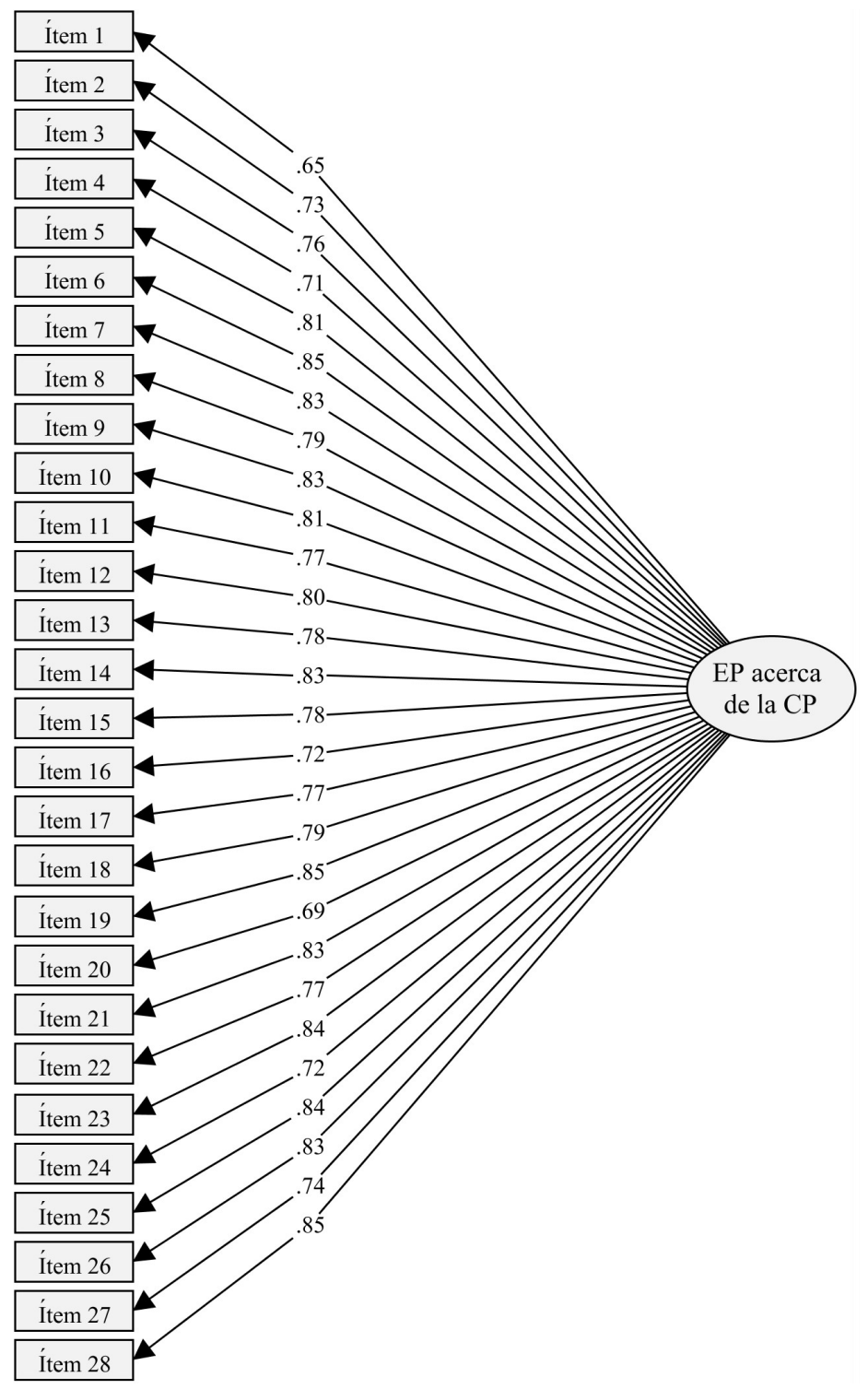

Figura 1. Estructura factorial versión final de la escala 
Tabla 5.

Índices de ajuste para el modelo unifactorial de la escala de expectativas parentales acerca de la prosocialidad de los hijos.

\begin{tabular}{cccccc}
\hline \multicolumn{6}{l}{ Índices de ajuste } \\
\hline $\mathbf{X}^{2 / \boldsymbol{g} I}$ & NFI & NNFI & CFI & IFI & RMSEA [IC] \\
\hline $2.26^{* * *}$ & .98 & .98 & .99 & .99 & .07 [.069-.083] \\
\hline
\end{tabular}

Nota: $\mathrm{x} 2 / \mathrm{gl}=$ chi cuadrada sobre los grados de libertad; ${ }^{\star \star \star} p<.01 ; \mathrm{NFI}=$ Índice de ajuste normalizado; NNFI = Índice de ajuste no normalizado; $\mathrm{CFI}=$ Índice de bondad de ajuste comparativo; IFI = Índice de ajuste incremental; RMSEA = Error de aproximación de la media cuadrática; IC $90 \%=$ Intervalos de confianza al $90 \%$

Finalmente, a partir de los coeficientes estandarizados, se calculó la fiabilidad compuesta y la variancia media extractada (VME). El índice de fiabilidad compuesta, el cual indica el grado de consistencia de los indicadores con la medida del constructo latente, fue de .97, coincidiendo con el índice Omega obtenido del análisis realizado con la primera muestra de estudio. La variancia media extractada fue de $61 \%$, similar al porcentaje de variancia explicada obtenido en el AFE.

\section{DISCUSIÓN}

Una importante limitación para el estudio de las expectativas parentales en Argentina es el escaso número de pruebas psicométricas construidas o adaptadas para analizar esta temática, tanto desde la perspectiva de los hijos como de los padres. Consecuentemente, el presente trabajo tuvo por objetivo la construcción y estudio psicométrico preliminar de una escala que valora las expectativas parentales acerca de la prosocialidad de los hijos desde la perspectiva de los padres. El diseño de la escala se planteó a partir de la necesidad de un instrumento de evaluación psicológica que pueda ser utilizado en diversos ámbitos de aplicación (e. g., clínico, educativo, comunitario, entre otros).

En cuanto al funcionamiento de los ítems de la escala, se pudo observar que todos los reactivos que la componen fueron discriminativos, lo que indica que permiten diferenciar adecuadamente entre quienes presentan diferentes niveles de expectativas acerca de la prosocialidad de los hijos. Estos resultados fueron consistentes con los obtenidos a partir de los indices de homogeneidad corregido.

Con respecto a la validez de constructo factorial de la escala, inicialmente se estimó que podría evidenciarse más de un factor, dado que, tal como se ha mencionado, las conductas prosociales involucran diversos tipos de comportamientos y actitudes (Caprara, et al, 2005; Eisenberg, Fabes, \& Spinrad, 2006; Grant \& Dutton, 2012; Lemos \& Richaud de Minzi, 2014, 2014; Mestre Escrivá, et al., 2006; Roche Olivar, 1995; Samper García, 2014). O bien, se pensó que las expectativas parentales acerca de la conducta prosocial podrian agruparse en torno a los diferentes ámbitos en que los hijos las manifiestan (familiar, escolar o no especificado). No obstante, el AFE mostró un solo factor subyacente en el conjunto de reactivos analizados. Esta estructura unifactorial fue confirmada a través del AFC aplicado en una segunda muestra, con un ajuste satisfactorio del modelo propuesto. Una posible explicación a estos resultados es que la mayoria de las conductas prosociales están altamente relacionadas entre sí, como lo han mostrado otros 
estudios (Balabanian \& Lemos, 2018; Caprara \& Pastorelli, 1993) en los que también se evidenció una estructura factorial unidimensional. Por otra parte, la escala sobre expectativas de los adolescentes acerca de la reacción de los padres, respecto a las conductas prosociales de los hijos, también mostró que todas las conductas prosociales se agruparon en torno a un único factor (Wyatt \& Carlo, 2002). Al seleccionar los ítems con mayor saturación, se escogieron 28 reactivos, pertenecientes en su mayoría a un contexto general (no específicos del ámbito familiar o escolar, e.g. "Trate bien a los demás"). Se observaron, en menor medida, items referidos al contexto escolar (e.g. "Valore las ideas de sus compañeros"), y solo dos vinculados al contexto familiar. Es posible que esta distribución se deba a que inicialmente se partió de un mayor grupo de ítems que pueden manifestarse indistintamente en diferentes contextos, con la intención de privilegiar la representatividad de los diversos tipos de conducta prosocial. Si bien inicialmente se escogieron como ámbitos relevantes de manifestación de las conductas prosociales en esta etapa, el familiar y escolar, algunas conductas de la versión final de la escala, podrían expresarse en ambos contextos (familiar y escolar) y/o en otros no especificados (en la relación con vecinos y amigos del barrio, en actividades deportivas o artísticas, etc.), lo que permitiría generalizar la conducta a diferentes situaciones de la vida cotidiana sin limitarlas a un contexto en particular.

Con respecto a los tipos de comportamientos que predominaron en la versión definitiva, de mayor a menor fueron: presencia positiva, revalorización positiva del otro, dar y compartir, cooperar, consolar y cuidar (Balabanian \& Lemos, 2018; Caprara, et al., 2005; Roche Olivar, 1995). De este modo, la versión final de la escala permite valorar, principalmente, conductas que contribuyen a un clima psicológico de bienestar, solidaridad, disponibilidad para el servicio y unidad entre los pares, todos comportamientos vinculados a una presencia positiva para con otros y en beneficio del grupo de pares. Por ejemplo: "Integre a otros a su grupo de amigos". También tuvieron una gran representación las conductas de revalorización positiva del otro, que implican expresiones verbales para confirmar el valor de otras personas, a través de palabras de reconocimiento, simpatía, elogios. Por ejemplo: "Reconozca las cualidades de sus compañeros". Finalmente, algunos items que aluden a dar y compartir posesiones personales, por ejemplo: "Done su ropa en buen estado, no solo lo que le sobra"; junto con otros reactivos vinculados a actividades de cooperación que implican un bien común, por ejemplo: "Colabore haciendo silencio cuando la maestra habla"; y consolar y cuidar, por ejemplo: "Consuele a algún compañero que esté triste".

En relación con la confiabilidad de la escala, el coeficiente Omega y la fiabilidad compuesta, indicó una alta consistencia interna (Campo-Arias \& Oviedo, 2008), lo que corrobora la alta correlación observada entre los diferentes items de la escala.

En síntesis, los hallazgos obtenidos hasta el momento permitieron seleccionar un total de 28 items con adecuadas propiedades psicométricas para valorar las expectativas de padres, madres o cuidadores acerca de la prosocialidad de niños escolarizados.

En lo que respecta a las limitaciones del estudio, puede señalarse que la muestra no es representativa de toda la población argentina. En investigaciones posteriores debería procurarse una inclusión más equitativa de participantes de otras provincias o regiones del país, como así también de 
centros educativos de otros estratos sociales (i.e., estratos bajos, altos y zonas rurales). Otra limitación que debería tenerse en cuenta es el bajo número de padres que participaron, lo que posiblemente se deba a que estos asisten con menor frecuencia a las actividades escolares que las madres. En consecuencia, sería recomendable balancear en número a padres y madres; así en un próximo trabajo se podría estudiar la invarianza factorial en función del sexo de los progenitores o cuidadores.

Asimismo, en un futuro estudio se podrian sumar otras evidencias de validez, como, por ejemplo, evaluar la validez predictiva de la escala de expectativas parentales acerca de la prosocialidad de los hijos, sobre específicamente las conductas prosociales de los niños (Richaud de Minzi, Lemos \& Mesurado, 2011; Wyatt \& Carlo, 2002) También podrian sumarse evidencias de validez de constructo concurrente, poniendo a prueba la hipótesis de que las expectativas parentales se relacionan con los comportamientos de los padres. Es decir, que las expectativas de los cuidadores afectan su propia conducta prosocial, tal como han indicado algunos estudios (e.g, Miller-Loncar, Landry, Smith, \& Swank, 2000).

Finalmente, la contribución de esta escala podría resultar valiosa para diversas áreas de la psicología aplicada (i.e., clínica, educativa, entre otras). Especialmente, podría colaborar en el diseño y aplicación de programas de intervención para promover las conductas prosociales tanto en el ámbito familiar como escolar. Adicionalmente, se estima que podria aportar una herramienta de investigación útil para la comprensión de los procesos perceptuales que actúan como mediadores en la relación entre padres e hijos y pueden resultar predictores del desarrollo de un recurso socioemocional tan relevante como lo son las conductas prosociales en la niñez.

\section{REFERENCIAS}

Auné, S. E. \& Attorresi, H. F. (2017). Dimensionalidad de un test de conducta prosocial. Revista Evaluar, 17(1), 29-37. https://doi.org/10.35670/1667-4545.v17.n1.17072

Balabanian, C. \& Lemos, V. (2018). Desarrollo y estudio psicométrico de una escala para evaluar conducta prosocial en adolescentes. Revista Iberoamericana de Diagnóstico y Evaluación Psicológica, 48(3), 177188. https://doi.org/10.21865/RIDEP48.3.15

Balabanian, C., Lemos, V., \& Vargas Rubilar, J. (2015). Apego percibido y conducta prosocial en adolescentes. Revista Colombiana de Ciencias Sociales, 6(2), $25-35$. https://doi.org/10.21501/22161201.1515

Bandalos, D. \& Finney, S. (2010). Factor Analysis: Exploratory and Confirmatory. En G. R. Hancock \& R. O. Mueller (Eds.), Reviewer's guide to quantitative methods. Routledge: New York.

Bandura, A., Barbaranelli, C., Caprara, G. V., \& Pastorelli, C. (1996). Multifaceted impact of self-efficacy beliefs on academic functioning. Child Development, 67, 1206-1222. https://doi.org/10.2307/1131888

Baumrind, D. (1978). Parental disciplinary patterns and social competence in children. Youth and Society, 9, 239-276. https://doi.org/10.1177/0044118X7800900302

Bornstein, M. (2007). Positive parenting and positive characteristics and values in children. En J. Coleman, D. Roker, R. Engels, H. Stattin, \& M. Kerr (Eds.), What Can Parents of Adolescents Do? (pp. 259-284). New York: Wiley. https://doi.org/10.1002/9780470774113.ch11

Campo-Arias, A. \& Oviedo, H. C. (2008). Propiedades Psicométricas de una Escala: la Consistencia Interna. Revista de Salud Pública, 10(5), 831-839. https://doi.org/10.1590/S0124-00642008000500015

Caprara, G. V., Steca, P., Zelli, A., \& Capanna, C. (2005). A new scale for measuring adults' prosocialness. European Journal of Psychological Assessment, 21(2), 77-89. https://doi.org/10.1027/10155759.21.2.77

Caprara, G. V. \& Pastorelli, C. (1993). Early emotional instability, prosocial behavior, and aggression: some methodological aspects. European Journal of Personality, 7, 19-36. https://doi.org/10.1002/per.2410070103

Carmines, E. G. \& Zeller, R. A. (1979). Reliability and Validity Assessment (Vol. 17). Thousand Oaks, CA: Sage. https://doi.org/10.4135/9781412985642

Carpenter, D. M. (2008). Expectations, aspirations, and achievement among Latino students of immigrant families. Marriage and Family Review, 43, 164-185. https://doi.org/10.1080/01494920802013078

Dunfield, K. A. (2014). A construct divided: Prosocial behavior as helping, sharing, and comforting subtypes. 
Frontiers in Psychology, 5, 958. https://doi.org/10.3389/fpsyg.2014.00958

Eisenberg, N., Fabes, R. A., \& Spinrad, T. L. (2006). Prosocial Development. En N. Eisenberg, W. Damon, \& R. M. Lerner (Eds.), Handbook of child psychology: Social, emotional, and personality development (pp. 646-718). Hoboken, NJ, US: John Wiley \& Sons Inc. https://doi.org/10.1002/9780470147658.chpsy0311

Ferrando, P. J. \& Lorenzo-Seva, U. (2014). El análisis factorial exploratorio de los items: algunas consideraciones adicionales. Anales de psicología, 30(3), 1170-1175. https://doi.org/10.6018/analesps.30.3.199991

Goldenberg, C., Gallimore, R., Reese, L., \& Garnier, H. (2001). Cause or effect? A longitudinal study of immigrant Latino parents' aspirations and expectations, and their children's school performance. American Educational Research Journal, 38(3), 547-582. https://doi.org/10.3102/00028312038003547

Grant, A. \& Dutton, J. (2012). Beneficiary or benefactor: are people more prosocial when they reflect on receiving or giving? Psychological Science, 23(9), 1033-1039. https://doi.org/10.1177/0956797612439424

Hoyle, R. (2012). Handbook of structural equation modeling. New York: Guilford Press

Jöreskog, K. \& Sörbom, D. (1993). LISREL 8: Structural Equation Modeling with the SIMPLIS Command Language, Chicago, IL: Scientific Software International Inc.

Juang, L. P. \& Silbereisen, R. K. (2002). The relationship between adolescent academic capability beliefs, parenting and school grades. Journal of Adolescence, 25, 3 - 18 . https://doi.org/10.1006/jado.2001.0445

Kline, R. (2005). Principies and practice of estructural equation modeling (2da. Edición) Nueva York: Guilford.

Lemos, V. (2015). Estudio de la validez de constructo, convergente, factorial y predictiva, del cuestionario de conducta prosocial para niños de 9 a 12 años. En V. N. Lemos (Org.). Avances en investigación en recursos socio cognitivos y afectivo. Simposio presentado en la XV Reunión Nacional y IV Encuentro Internacional de la Asociación Argentina de Ciencias del Comportamiento. Tucumán, Argentina.

Lemos, V. \& Richaud de Minzi, M. C. (2014). Promotion of child prosocial behavior in the school context. En A. Castro Solano (Ed.), Positive Psychology in LatinAmerica (pp. 179-193). Netherlands: Springer. https://doi.org/10.1007/978-94-017-9035-2_10

Lorenzo-Seva, U., \& Ferrando, P. J. (2013). Factor 9.2: A comprehensive program for fitting exploratory and semiconfirmatory factor analysis and IRT models. Applied Psychological Measurement, 37(6), 497-498. https://doi.org/10.1177/0146621613487794

Martínez Arias, R., Hernández Lloreda, M. V., \& Hernández Lloreda, M. J. (2006). Psicometría. Madrid: Alianza.

McDonald, R. P. (1999). Test theory: A unified treatment. Mahwah: Lawrence Erlbaum Associates, Inc

Mestre Escrivá, M. V., Samper, P., Tur, A. M., Cortés, M. T., \& Nácher, M. J. (2006). Conducta prosocial y procesos psicológicos implicados: un estudio longitudinal en la adolescencia. Revista Mexicana de Psicología, 23(2), 203-216.

Mestre Escrivá, M. V.,Tur, A., Samper, A., Nácher, M. J., \& Cortés, M. T. (2007). Estilos de crianza en la adolescencia y su relación con el comportamiento prosocial. Revista Latinoamericana de Psicología, 39(2), 211-225.

Miller-Loncar, C. L., Landry, S. H., Smith, K. E., \& Swank, P. R. (2000). The influence of complexity of maternal thoughts on sensitive parenting and children's social responsiveness. Journal of Applied Developmental Psychology, 21(3), 335-356. https://doi.org/10.1016/S0193-3973(00)00044-7

Montero, I. \& León, O. (2007). A guide for naming research studies in Psychology. International Journal of Clinical and Health Psychology, 7(3), 847-862.

Muthén, B. \& Kaplan D. (1985). A comparison of some methodologies for the factor analysis of non-normal Likert variables. British Journal of Mathematical and Statistical Psychology, 38, 171-189. https://doi.org/10.1111/j.2044-8317.1985.tb00832.x

Oishi, S. \& Sullivan, H. W. (2005). The mediating role of parental expectations in culture and wellbeing. Journal of Personality, 73(5), 1267-1294. https://doi.org/10.1111/j.1467-6494.2005.00349.x

Oliva Delgado, A. (2011). Apego en la adolescencia. Acción Psicológica, 8(2), 55-65. https://doi.org/10.5944/ap.8.2.190

Ortiz, M. J., Apodaka, P., Etxeberria, I., Ezeiza, A., Fuentes, M. J., \& López, F. (1993). Algunos predictores de la conducta prosocial-altruista en la infancia: empatía, toma de perspectiva, apego, modelos parentales, disciplina familiar e imagen del ser humano. Revista de Psicología Social, 8(1), 83-98. https://doi.org/10.1080/02134748.1993.10821671

Otzen, T. \& Manterola, C. (2017). Técnicas de muestreo sobre una población a estudio. International Journal of Morphology, 35(1), 227-232. https://doi.org/10.4067/S0717-95022017000100037

Redondo Pacheco, J., Rueda Rueda, S., \& Amado Vega, C. (2013). Conducta prosocial: Una alternativa a las conductas agresivas. InvestigiumIre: Ciencias Sociales y Humanas, 4(1), 234-247.

Richaud de Minzi, M. C. (2009). Influencia del Modelado de los Padres sobre el desarrollo del razonamiento prosocial en los/las niños/as. Interamerican Journal of Psychology, 43, 187-198.

Richaud de Minzi, M.C., Lemos, V., \& Mesurado, B. (2011). Relaciones entre percepción que tienen los niños de los estilos de relación y de la empatía de los padres y la conducta prosocial en la niñez media y tardia. Avances en psicología latinoamericana, 29(2), 330-343.

Richaud de Minzi, M. C., Mesurado, B., Samper, P., Llorca, A. Lemos, V., \& Tur, A. (2013). Estilos parentales, inestabilidad emocional y agresividad en niños de nivel socioeconómico bajo en Argentina y España. Ansiedad y Estrés, 19(1), 53-69.

Roche Olivar, R. (1995). Psicología y educación para la prosocialidad. Buenos Aires: Ciudad Nueva.

Roche Olivar, R. (1998). El uso educativo de la televisión como optimizadora de la prosocialidad. 
Psychosocial Intervention, 7(3), 363-378.

Samper García, P. (2014). Diferentes tendencias prosociales: El papel de las emociones. Revista mexicana de Investigación en Psicología, 6(2), 177-185.

Sánchez-Queija, I. \& Oliva, (2003). Vínculos de apego con los padres y relaciones con los iguales durante la adolescencia. Revista de Psicologia Social, 18(1), 71-86. https://doi.org/10.1174/02134740360521796

Squires, J., Bricker, D., \& Twombly, E. (2013). The ASQ:SE User's Guide. Baltimore: Paul H. Brookes Publishing.

Tomás, J. M. \& Oliver, A. (1998). Efectos de formato de respuesta y método de estimación en análisis factorial confirmatorio. Psicothema, 10(1), 197-208.

Vargas Rubilar, J. \& Richaud M.C. (2018). Childhood Parenting: Main approaches and aspects analyzed from psychology. En C. Garcia (Ed.), Research on Hispanic Psychology, Vol. 1 (pp. 241-276). New York: Nova Science Publishers, Inc.

Vargas Rubilar, J., Oros, L., \& Lemos, V. (octubre, 2016). Efectos de la parentalidad sobre las emociones y los comportamientos sociales infantiles: una sintesis de estudios argentinos. En M. V. Mestre (Org.). Crianza y conducta en la infancia y adolescencia: El rol de las emociones. Simposio presentado en el $2^{\circ}$ Congreso Latinoamericano para el avance de la ciencia psicológica. Buenos Aires, Argentina.

Ventura-León, J. L. \& Caycho-Rodríguez, T. (2017). El coeficiente omega: un método alternativo para la estimación de la confiabilidad. Revista Latinoamericana de Ciencias Sociales, Niñez y Juventud, 15(1), 625-627.

Warneken, F. \& Tomasello, M. (2009). The roots of human altruism. British Journal of Psychological Society, 100, 455-471. https://doi.org/10.1348/000712608X379061

Wyatt, J. M. \& Carlo, G. (2002). What will my parents think? Relations among adolescents' expected parental reactions, prosocial moral reasoning, and prosocial and antisocial behaviors. Journal of Adolescent Research, 17(6), 646-666. https://doi.org/10.1177/074355802237468

Yamamoto, Y. \& Holloway, S. D. (2010). Parental Expectations and Children's Academic Performance in Sociocultural Context. Educational Psychology Review, 22(3), 189-214. https://doi.org/10.1007/s10648-010-9121-z

Recibido 02-10-2018 | Aceptado 30-04-2019

Este trabajo se encuentra bajo una Licencia Creative Commons Atribución 4.0 Internacional que permite a terceros utilizar lo publicado siempre que se dé el crédito pertinente a los autores y a Psicodebate 